\title{
Comparación de tres cuestionarios pronósticos de riesgo para síndrome obstructivo de apnea e hipopnea de sueño en adultos
}

Ricardo Andres Escobar Baigorria 2,b; Franca Maraís Sifuentes Peracchio* 2,b; Félix Llanos-Tejada ${ }^{1,2, a}$

RESUMEN

Objetivo: Determinar las diferencias en el pronóstico del riesgo de presentar el síndrome obstructivo de apnea/hipopnea del sueño (SOAHS) al comparar los resultados de los cuestionarios de Berlín (CB), STOP-Bang (SB) y la Escala de somnolencia de Epworth (ESE) en pacientes internados en el Hospital Nacional Dos de Mayo durante el periodo 2019-2020.

Materiales y métodos: Estudio clínico cuantitativo, observacional, descriptivo de pruebas diagnósticas, prospectivo y transversal. Se incluyó una muestra de 304 pacientes mayores de 18 años. Los resultados se presentan con frecuencias, medidas de tendencia central y de dispersión. Las variables se asociaron mediante la prueba de X². Se midió la sensibilidad y especificidad del CB y ESE respecto a SB. El valor de $p$ menor de 0,05 se consideró como significativo.

Resultados: El promedio de edad fue de 52,89 \pm 15,97 años, con predominio de los adultos menores de 50 años (40,50 \%). El $60,50 \%$ de los participantes fueron de sexo masculino. El promedio del IMC fue 30,74 $\pm 6,02 \mathrm{~kg} / \mathrm{m}^{2}$ y la categoría más numerosa fue el sobrepeso (43,40\%). El 42,80 \% tenía hipertensión. Según el SB, CB y la ESE, el 77,00 \%, 62,50 \% y 60,50 \% tenían alto riesgo de presentar el SOAHS, respectivamente. Los cuestionarios mostraron una asociación entre edad, sexo, diagnóstico nutricional e hipertensión arterial con un elevado riesgo de presentar SOAHS $(p<0,05)$. Existe una débil correlación entre SB y CB (Kappa: 0,39) y SB y ESE (Kappa: 0,33).

Conclusiones: Los cuestionarios son herramientas útiles con el potencial de pronosticar el riesgo de SOAHS. Presentan una correlación significativa, pero débil, entre su pronóstico de riesgo del síndrome. Sin embargo, deben ser considerados como pruebas de detección rápida y confiable.

Palabras clave: Diagnóstico; Síndromes de la apnea del sueño; Encuestas y cuestionarios; Polisomnografía (Fuente: DeCS BIREME).

\section{A comparison of three questionnaires to predict the risk of obstructive sleep apnea and hypopnea syndrome in adults}

ABSTRACT

Objective: To determine the differences in the prediction of the risk of developing obstructive sleep apnea-hypopnea syndrome (OSAHS) by comparing the results of the Berlin Questionnaire (BQ), the STOP-Bang (SB) Questionnaire and the Epworth Sleepiness Scale (ESS) in patients admitted to the Hospital Nacional Dos de Mayo from 2019 to 2020.

Materials and methods: A quantitative, observational, descriptive, prospective and cross-sectional clinical study of diagnostic tests. A sample of 304 patients older than 18 years was included in the research. The results are presented with frequencies, measures of central tendency and dispersion. The variables were associated using the chi-square test. The sensitivity and specificity of the BQ and ESS were measured against the SB Questionnaire. A p value less than 0.05 was considered as statistically significant.

Results: The average age of the study population was $52.89 \pm 15.97$ years and adults under 50 years of age (40.5 \%) prevailed. Sixty point five percent $(60.5 \%)$ of the participants were males. The average BMI was $30.74 \pm 6.02 \mathrm{~kg} / \mathrm{m}^{2}, \mathrm{with}$ the overweight category being the most numerous (43.40\%). Forty-two point eight percent (42.8\%) of the participants had hypertension. According to the SB Questionnaire, BQ and ESS, 77.0 \%, $62.5 \%$ and $60.5 \%$ had a high risk of developing OSAHS, respectively. The questionnaires showed an association between age, sex, nutritional diagnosis and arterial hypertension, and a high risk of developing OSAHS $(p<0.05)$. There is a weak correlation between the SB Questionnaire and BQ (Kappa: 0.39), and between the SB Questionnaire and ESS (Kappa: 0.33).

Conclusions: The abovementioned questionnaires are useful tools to predict the risk of developing OSAHS. They show a significant but weak correlation between them. However, they should be considered as rapid and reliable screening tests.

Keywords: Diagnosis; Sleep apnea syndromes; Surveys and questionnaires; Polysomnography (Source: MeSH NLM).

1 Hospital Nacional Dos de Mayo. Lima, Perú.

2 Universidad de San Martín de Porres, Facultad de Medicina Humana. Lima, Perú.

a Médico Neumólogo.

b Estudiante de pregrado.

*Autor corresponsal.

Horiz Med (Lima) 2021; 21(2):e1347 


\section{INTRODUCCIÓN}

El síndrome obstructivo de apnea e hipopnea de sueño (SOAHS) es un cuadro caracterizado por somnolencia diurna excesiva, fatiga, trastornos cognitivo-conceptuales, cardíacos, metabólicos, inflamatorios o respiratorios producido por la interrupción repetida del flujo de aire en la vía aérea superior durante el sueño ${ }^{(1)}$.

La apnea del sueño se define por la presencia de, al menos, cinco episodios de apnea por hora, de 10 o Más segundos, acompañados por desaturación de oxígeno e interrupción del sueño. Los factores de riesgo para SOAHS incluyen obesidad, características craneofaciales, como la micrognatia, antecedentes familiares de este cuadro y el sexo masculino. Además, este síndrome ha sido relacionado a enfermedades sistémicas como hipotiroidismo y acromegalia (2).

El SOAHS es la enfermedad respiratoria más común relacionada al sueño, es prevalente en el sexo masculino y su frecuencia aumenta con la edad. No obstante, puede presentarse también en mujeres y niños. La diferencia en la incidencia entre sexos disminuye en la posmenopausia ${ }^{(3-5)}$.

Se estima que más de 900 millones de adultos menores de 70 años en el mundo presentan síntomas de SOAHS que van de leves a severos. Diversos estudios han reportado elevación en la prevalencia del SOAHS que puede atribuirse a la tendencia del incremento de sobrepeso y obesidad en sus poblaciones y al aumento del diagnóstico de este síndrome ${ }^{(6-8)}$.

El diagnóstico oportuno y accesible del SOAHS es importante debido a que este causa hipoxemia, hipercapnia, taquicardia, secreción de adrenalina, noradrenalina y cortisol durante el sueño, lo que altera de forma directa el endotelio y produce un incremento en el riesgo de enfermedades cardiovasculares, tales como hipertensión arterial, arteriopatía coronaria, insuficiencia cardiaca, apoplejía y arritmias. Además, repercute en el sistema nervioso central y lo convierte en un importante factor de riesgo cerebrovascular independiente e incrementa la frecuencia y severidad de las enfermedades neurológicas como epilepsia y síndromes demenciales ${ }^{(2,9-12)}$.

En el Perú existen pocas investigaciones y datos epidemiológicos sobre su prevalencia a pesar de su importancia y el aumento de la morbimortalidad por enfermedades cardiovasculares en los últimos 30 años, las cuales representan el $15 \%$ de las causas de mortalidad prematura en Lima y Callao ${ }^{(13)}$.

La polisomnografía (PSG) es el método estándar para establecer el diagnóstico definitivo del SOAHS. Sin embargo, es muy costosa y poco accesible en el Perú. Es por esto que se han desarrollado herramientas más asequibles que permiten detectar a los pacientes en riesgo de presentar este síndrome, tales como el Cuestionario de Berlín (CB), STOP-Bang (SB) y la Escala de somnolencia de Epworth (ESE) ${ }^{(14)}$.

Existen numerosos estudios en todo el mundo que analizan la confiabilidad de las diversas herramientas diagnósticas con la PSG, pero son pocos los que las comparan entre sí. Se ha relacionado el valor predictivo del CB, SB y la ESE en pacientes con SOAHS en un grupo de roncadores atendidos en una clínica de sueño, y se encontró que 86,92 y $46 \%$ de los casos fueron clasificados como de alto riesgo para SOAHS, respectivamente, y el SB mostró la mayor sensibilidad (S) para predecir SOAHS $(95 \%)^{(15)}$.

En este estudio se busca determinar las diferencias en el pronóstico del riesgo de presentar el SOAHS comparando los resultados de los $C B$, SB y la ESE en pacientes mayores de 18 años, con factores de riesgo y que estaban internados en el Hospital Nacional Dos de Mayo durante el periodo 2019-2020.

\section{MATERIALES Y MÉTODOS}

\section{Diseño y población de estudio}

Estudio clínico cuantitativo, observacional, descriptivo de pruebas diagnósticas, prospectivo y transversal. La población universo incluyó a una muestra de 304 pacientes mayores de 18 años del Hospital Nacional Dos de Mayo, internados en el servicio de Medicina Interna, que contaban con factores de riesgo para SOAHS.

\section{Variables y mediciones}

Las características clínicas asociadas al riesgo de SOAHS evaluadas en este estudio fueron la edad, el sexo, el diagnóstico nutricional según el IMC y la hipertensión arterial. Para obtener estos datos se emplearon los cuestionarios CB, SB y ESE.

El CB se basa en identificar los factores de riesgo para determinar la presencia del SOAHS, las preguntas representan los más importantes (ronquido, somnolencia diurna, cansancio, obesidad e hipertensión arterial) ${ }^{(16)}$.

El cuestionario SB toma su nombre de sus siglas en inglés: $S$ de snore (ronquido), $T$ de tired (cansancio o somnolencia), $O$ de observed apneas (apneas observadas), $\mathrm{P}$ de pressure (HTA), B de BMI (índice de masa corporal,en inglés Body mass index $>35 \mathrm{~kg} / \mathrm{m}^{2}$ ), A de age (edad $\left.>50\right)$, N de neck (circunferencia del cuello $>40 \mathrm{~cm}$ ) y G de gender (sexo masculino) ${ }^{(17)}$.

La ESE es un instrumento que evalúa la somnolencia de manera subjetiva en ocho situaciones cotidianas diferentes ${ }^{(18)}$. 


\section{Análisis estadístico}

Los datos obtenidos de los CB, SB y ESE fueron ingresados en una matriz de sistematización construida en el software Microsoft Office Excel 2016. Se utilizó el software IBM SPSS versión 25 para realizar los cuadros estadísticos.

En el caso de las variables cuantitativas, los resultados se presentan con medidas de tendencia central y de dispersión; y en el caso de las variables cualitativas, como frecuencias. Las variables se asociaron mediante la prueba de $X^{2}$. Se midió la sensibilidad y especificidad del cuestionario $C B$ y ESE respecto a SB y el valor de $p$ menor a 0,05 se consideró significativo.

\section{Consideraciones éticas}

El protocolo de investigación y el consentimiento informado fueron aprobados por el Comité de Ética de la Facultad de Medicina Humana de la Universidad de San Martín de Porres y el Comité de Ética del Hospital Nacional Dos de Mayo.

\section{RESULTADOS}

Evaluamos 304 pacientes con factores de riesgo para SOAHS que estaban hospitalizados en el Servicio de Medicina Interna en el Hospital Nacional Dos de Mayo, durante el periodo 2019-2020. El promedio de edad fue de 52,89 $\pm 15,97$ años, con un rango entre 19 a 89 años, con un predominio de adultos menores de 50 años (40,50\%). Además, el 60,50\% de los participantes fueron de sexo masculino. Respecto al diagnóstico nutricional, el promedio del IMC fue $30,74 \pm 6,02 \mathrm{~kg} / \mathrm{m}^{2}$, la categoría más frecuente fue el sobrepeso $(43,40 \%)$, seguido de obesidad tipo I (28,9\%). Asimismo, el $42,80 \%$ de los pacientes tenía hipertensión. Según el SB, CB y la ESE, el 77,00 \%, 62,50 \% y $60,50 \%$ de los pacientes tenían alto riesgo de presentar el SOAHS, respectivamente (Tabla 1 ).

Tabla 1. Características de los participantes

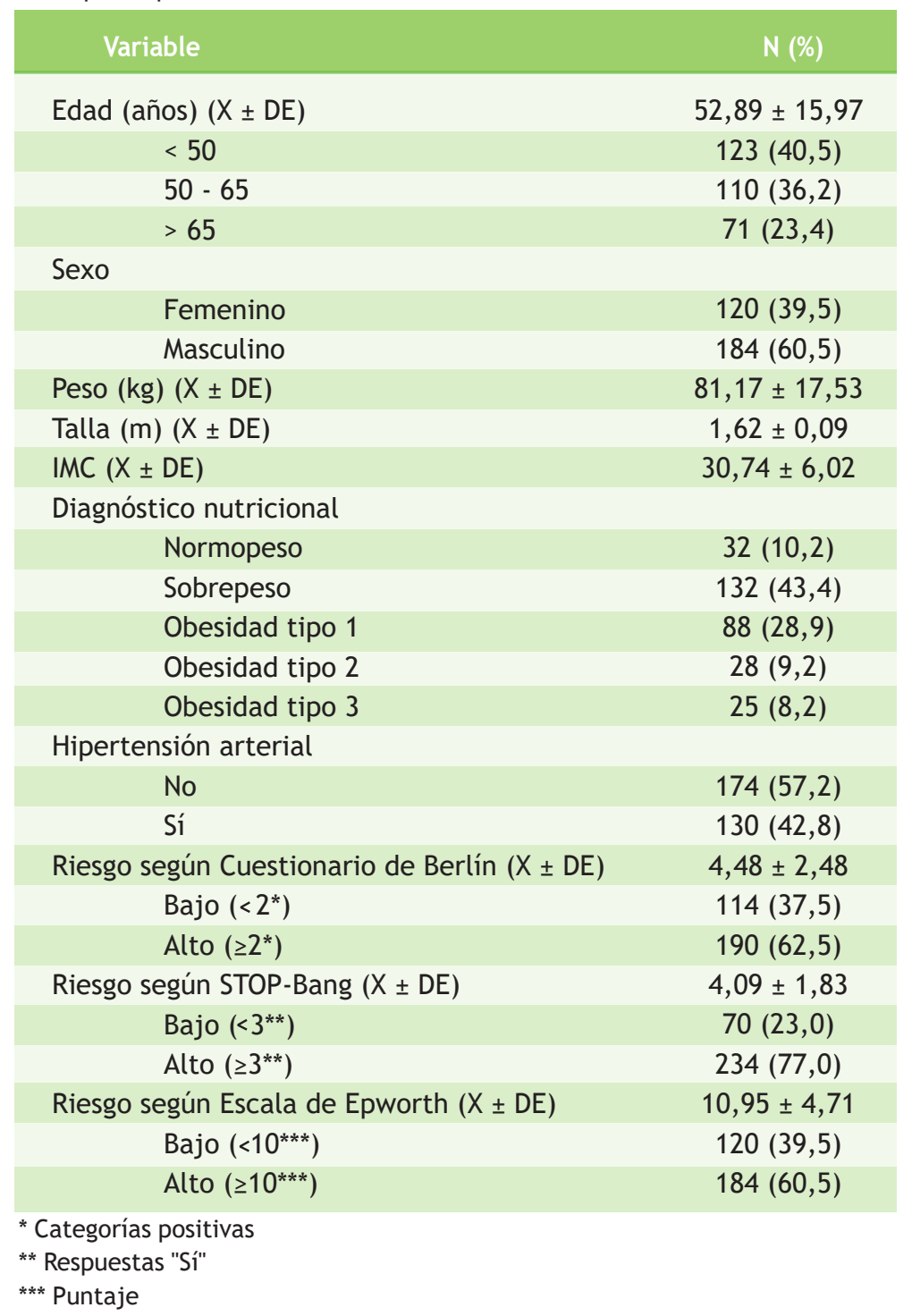


El riesgo de presentar SOAHS fue mayor en pacientes de 50 a 65 años, según los tres cuestionarios $(p<0,05)$. Los pacientes de sexo masculino tuvieron mayor riesgo de presentar SOAHS según CB y SB $(p<0,05)$. El CB encontró que había mayor riesgo de presentar SOAHS en los pacientes con obesidad tipo 1 y sobrepeso $(p<0,05)$, hallazgos que concuerdan con los resultados de $\mathrm{SB}(p<0,05)$. En contraste, la ESE no pudo mostrar una diferencia estadísticamente significativa con respecto al diagnóstico nutricional de los pacientes. Por último, los tres cuestionarios hallaron una asociación entre hipertensión arterial y un elevado riesgo de presentar SOAHS $(p<0,05)$ (Tabla 2$)$.

Tabla 2. Asociación entre el nivel de riesgo según Cuestionario de Berlín, STOP-Bang y Escala de Somnolencia de Epworth y otras variables de los participantes

\begin{tabular}{|c|c|c|c|c|c|c|c|c|c|c|c|c|c|c|c|}
\hline & \multicolumn{4}{|c|}{ Cuestionario de Berlín } & & \multicolumn{4}{|c|}{ Stop-BANG } & \multicolumn{6}{|c|}{ Escala de Epworth } \\
\hline & Riesg & o Bajo & Riess & go Alto & Valor de & Riesg & so Bajo & Riesg & go Alto & Valor de & Riesg & o Bajo & Ries! & go Alto & Valor de \\
\hline & $n$ & $\%$ & $\mathrm{n}$ & $\%$ & $p$ & $n$ & $\%$ & $n$ & $\%$ & p & $n$ & $\%$ & $\mathrm{n}$ & $\%$ & $p$ \\
\hline \multicolumn{16}{|l|}{ Edad (años) } \\
\hline$<50$ & 63 & 53,3 & 60 & 31,6 & $<0,001$ & 56 & 80,0 & 67 & 28,6 & $<0,001$ & 60 & 50,0 & 63 & 34,2 & 0,013 \\
\hline $50-65$ & 33 & 28,9 & 77 & 40,5 & & 11 & 15,7 & 99 & 42,3 & & 33 & 27,3 & 77 & 41,8 & \\
\hline$>65$ & 18 & 15,8 & 53 & 27,9 & & 3 & 4,3 & 68 & 29,1 & & 27 & 22,5 & 44 & 23,9 & \\
\hline \multicolumn{16}{|l|}{ Sexo } \\
\hline Femenino & 37 & 32,5 & 83 & 43,7 & 0,053 & 38 & 54,3 & 82 & 35,0 & 0,004 & 54 & 45,0 & 66 & 35,9 & 0,111 \\
\hline Masculino & 77 & 67,5 & 107 & 56,3 & & 32 & 45,7 & 152 & 65,0 & & 66 & 55,0 & 118 & 64,1 & \\
\hline \multicolumn{16}{|c|}{ Diagnóstico nutricional } \\
\hline Normopeso & 13 & 11,4 & 18 & 9,5 & $<0,001$ & 4 & 5,7 & 27 & 11,5 & $<0,001$ & 14 & 11,7 & 17 & 9,2 & 0,477 \\
\hline Sobrepeso & 77 & 67,5 & 55 & 28,9 & & 46 & 65,7 & 86 & 36,8 & & 56 & 46,7 & 76 & 41,3 & \\
\hline Obesidad tipo 1 & 21 & 18,4 & 67 & 35,3 & & 19 & 27,1 & 69 & 29,5 & & 34 & 28,3 & 54 & 29,3 & \\
\hline Obesidad tipo 2 & 1 & 0,9 & 27 & 14,2 & & 1 & 1,4 & 27 & 11,5 & & 7 & 5,8 & 21 & 11,4 & \\
\hline Obesidad tipo 3 & 2 & 1,8 & 23 & 12,1 & & 0 & 0,0 & 25 & 10,7 & & 9 & 7,5 & 16 & 8,7 & \\
\hline \multicolumn{16}{|c|}{ Hipertensión arterial } \\
\hline No & 85 & 74,6 & 89 & 46,8 & $<0,001$ & 60 & 85,7 & 114 & 48,7 & $<0,001$ & 79 & 65,8 & 95 & 51,6 & 0,014 \\
\hline Sí & 29 & 25,4 & 101 & 53,2 & & 10 & 14,3 & 120 & 51,3 & & 41 & 34,2 & 89 & 48,4 & \\
\hline
\end{tabular}

Se observa que existe una débil correlación entre SB y CB (Kappa: 0,39) y SB y ESE (Kappa: 0,33) (Tabla 3).

Tabla 3. Resultados de riesgo de SOAHS de Cuestionario de Berlín y Escala de Somnolencia de Epworth en comparación con STOP-Bang

\begin{tabular}{|c|c|c|c|c|c|c|c|}
\hline \multirow[b]{3}{*}{ Variable } & \multicolumn{7}{|c|}{ STOP-Bang } \\
\hline & & \multicolumn{2}{|c|}{ Riesgo Bajo } & \multicolumn{2}{|c|}{ Riesgo Alto } & \multirow{2}{*}{$\begin{array}{c}\text { Valor de } \\
p\end{array}$} & \multirow[t]{2}{*}{ Kappa } \\
\hline & & N & $(\%)$ & N & $(\%)$ & & \\
\hline \multirow[t]{2}{*}{ Cuestionario de Berlín } & Riesgo bajo & 52 & $74,3^{* *}$ & 62 & 26,5 & $<0,001$ & 0,392 \\
\hline & Riesgo alto & 18 & 25,7 & 172 & $73,5^{*}$ & & \\
\hline \multirow[t]{2}{*}{ Escala de somnolencia de Epworth } & Riesgo bajo & 50 & $71,4^{* *}$ & 70 & 29,9 & $<0,001$ & 0,332 \\
\hline & Riesgo alto & 20 & 28,6 & 164 & $70,1^{*}$ & & \\
\hline
\end{tabular}

* Sensibilidad de la prueba con respecto a cuestionario de STOP-Bang

** Especificidad de la prueba con respecto a cuestionario de STOP-Bang 


\section{DISCUSIÓN}

Encontramos una correlación entre los resultados obtenidos por los cuestionarios $\mathrm{CB}, \mathrm{SB}$, y ESE en una población con factores de riesgo para presentar SOAHS. El SB se tomó como referencia, porque es el más sensible de los tres cuestionarios para pronosticar el riesgo de tener SOAHS; se evidenció que esta correlación es débil, sobre todo entre SB y ESE. Esto posiciona a la ESE como la prueba diagnóstica con menor sensibilidad lo que coincide con los resultados de Saldías Peñafiel et al. ${ }^{(15)}$.

Los tres cuestionarios coincidieron en que la población con mayor riesgo de SOAHS tenía entre 50 a 65 años, seguida por los menores de 50 años y la población mayor de 65 es la que tenía menor riesgo de presentarlo. Este resultado es similar al de Pachauri Vallenas (Arequipa, Perú) que, con el CB, evidenció que de una población de 193 adultos, la mayor proporción de participantes con SOAHS tenía menos de 50 años y que el grupo con más de 66 años tenía la menor proporción de este síndrome, lo que se confirmó con polisomnografía ${ }^{(19) .}$

Los cuestionarios $S B$ y CB evidenciaron una asociación entre el sexo masculino y mayor riesgo de presentar SOAHS, pero la ESE no mostró una diferencia estadísticamente significativa para esta variable. Estos hallazgos concuerdan con el estudio realizado por Chung, F. en Canadá (2008), que encontró una $S B$ aumentada en los pacientes de sexo masculino para el diagnóstico del SOAHS, en conjunto con otras variables que componen los criterios de dicho cuestionario ${ }^{(20)}$.

Un estudio peruano muestra que la hipertensión arterial y obesidad tuvieron una asociación estadísticamente significativa con este síndrome (21). Esto concuerda con nuestra investigación, en la que el diagnóstico nutricional, según el $\mathrm{CB}$, mostró un mayor riesgo de presentar SOAHS en los pacientes con obesidad tipo 1 , lo que se opone a los resultados hallados por el SB, que plantea que los pacientes con sobrepeso tuvieron mayor riesgo, al igual que en la ESE. A pesar de que este último no encontrará una diferencia estadísticamente significativa en cuanto a dicha variable.

Finalmente, evidenciamos una mayor incidencia de hipertensión arterial en pacientes con alto riesgo de SOAHS, según los tres cuestionarios. Lo mismo concluyeron Saldías Peñafiel et al. en Chile, que consideraron a la hipertensión arterial como uno de los principales factores de riesgo para SOAHS tras estudiar el valor predictivo positivo de diversos cuestionarios para este síndrome, incluyendo a SB, CB y ESE. Asimismo, Gómez Abanto, J., en su estudio en Perú, determinó que la hipertensión arterial, así como otras enfermedades cardiovasculares, tenían una asociación estadísticamente significativa con este síndrome, que se establece como factor de riesgo para ellas ${ }^{(15,21)}$.
Una de las limitaciones de este estudio radica en no haber realizado el PSG para el diagnóstico, debido al escaso número y difícil acceso de esta prueba en el país. De lo contrario, se hubiera ampliado la investigación para confirmar el diagnóstico en los pacientes de alto riesgo y evaluar la sensibilidad y especificidad de las tres pruebas diagnósticas estudiadas respecto al estándar de oro para el diagnóstico de este síndrome. Por este motivo, solo se contó con predicciones diagnósticas y recomendamos que estudios posteriores consideren este aspecto en su diseño.

La muestra no pudo ser ampliada por la emergencia sanitaria ocasionada por la pandemia del SARS-CoV-2 y su llegada a Lima, Perú. El acceso a los hospitales al inicio fue limitado y luego restringido. Esto limitó las intenciones futuras de profundizar el estudio en los pacientes con alto riesgo a través de la prueba de poligrafía respiratoria.

Al tratarse de cuestionarios autoadministrados, se pueden presentar sesgos como el de fatiga, recuerdo, pesimismo y optimismo, a pesar de haber sido validados internacionalmente; para contrarrestar esta posibilidad, los investigadores estuvieron presentes durante el llenado de las encuestas para asegurar una correcta ejecución de este procediemiento.

Concluimos que estos tres cuestionarios son herramientas útiles con el potencial de pronosticar el SOAHS en una población con factores de riesgo, ya que presentan una correlación significativa, aunque débil. Sin embargo, deben ser considerados como pruebas de detección rápida y confiable que permitiría a los médicos conocer, durante las visitas clínicas iniciales, la posibilidad de algunos pacientes de desarrollar SOAHS, y determinar a aquellos con alto riesgo que necesitan una evaluación adicional. Sugerimos emplear el cuestionario STOP-Bang por su sensibilidad y por ser un método sencillo y fácil de recordar por su acrónimo. Además, recomendamos el uso de la ESE como complemento para evidenciar la alteración del sueño producida por este síndrome.

Contribuciones de los autores: Ricardo Andrés Escobar Baigorria y Franca Maraís Sifuentes Peracchio han participado en cada etapa del estudio: la elaboración del protocolo de investigación, recolección de datos, análisis e interpretación de los resultados y redacción del informe final. Félix Konrad Llanos Tejada realizó una revisión crítica del manuscrito, asesoría estadística y metodológica.

Fuentes de financiamiento: Este artículo ha sido financiado por los autores.

Conflictos de interés: Los autores declaran no tener ningún conflicto de interés. 


\section{REFERENCIAS BIBLIOGRAFICAS}

1. Durán-Cantolla J, González-Mangado N. Consenso Nacional sobre el síndrome de apneas-Hipopneas del sueño (SAHS)-Resumen. Arch Bronconeumol. 2005; 41(4): 7-9.

2. Kasper DL, Fauci AS, Hauser SL, Longo DL, Jameson JL, Loscalzo J. Harrison. Principios de Medicina Interna. 19na ed. México D. F.: McGraw-Hill Interamericana; 2017. pp. 745-6.

3. Young T, Palta M, Dempsey J, Skatrud J, Weber S, Badr S. The occurrence of sleep-disordered breathing among middle-aged adults. N Engl J Med. 1993; 328(17): 1230-5.

4. Bixler EO, Vgontzas AN, Ten Have T, Tyson K, Kales A. Effects of age on sleep apnea in men: I. Prevalence and severity. Am J Respir Crit Care Med. 1998; 157(1): 144-8.

5. Bixler EO, Vgontzas AN, Lin HM, Ten Have T, Rein J, Vela-Bueno A, et al. Prevalence of sleep-disordered breathing in women: effects of gender. Am J Respir Crit Care Med. 2001; 163(3 Pt 1): 608-13.

6. Benjafield AV, Ayas NT, Eastwood PR, Heinzer R, Ip MSM, Morrell MJ, et al. Estimation of the global prevalence and burden of obstructive sleep apnoea: a literature-based analysis. Lancet Respir Med. 2019; 7(8): 687-9.

7. Peppard PE, Young T, Palta M, Skatrud J. Prospective study of the association between sleep-disordered breathing and hypertension. $\mathrm{N}$ Engl J Med. 2000; 342(19): 1378-84.

8. Lechner M, Breeze CE, Ohayon MM, Kotecha B. Snoring and breathing pauses during sleep: interview survey of a United Kingdom population sample reveals a significant increase in the rates of sleep apnoea and obesity over the last 20 years - data from the UK sleep survey. Sleep Med. 2019; 54: 250-6.

9. Friedman O, Logan AG. The price of obstructive sleep apneahypopnea: hypertension and other ill effects. Am J Hypertens 2009; 22(5): 474-83.

10. Grossman S, Porth CM. Porth. Fisiopatología. Alteraciones de la salud. Conceptos básicos. 9na ed. L'Hospitalet de Llobregat, Barcelona: Wolter Kluwer; 2015. pp.537-8.

11. Barón A, Páez-Moya S. Cardiovascular repercussions of obstructive sleep apnea-hypopnea syndrome (OSAHS). Rev Fac Med. 2017; 65(Supl 1): 39-46.

12. Venegas-Mariño M. Neurological implications of obstructive sleep apnea-hypopnea syndrome (OSAHS). Rev Fac Med. 2017; 65(Supl 1): 47-9.

13. Hernández-Vásquez A, Díaz-Seijas D, Espinoza-Alva D, Vilcarromero S. Análisis espacial de la mortalidad distrital por enfermedades cardiovasculares en las provincias de Lima y Callao. Rev Peru Med Exp Salud Pública. 2016; 33(1): 185.

14. Academia Estadounidense de Medicina del Sueño. Manual de la AASM para la puntuación del sueño y eventos asociados: reglas, terminología y especificaciones técnicas, versión 2.6. Academia Estadounidense de Medicina del Sueño; 2020.

15. Saldías Peñafiel F, Gassmann Poniachik J, Canelo López A, Uribe Monasterio J, Díaz Patiño O, Saldías Peñafiel F, et al. Accuracy of sleep questionnaires for obstructive sleep apnea syndrome screening. Rev Médica Chile. 2018; 146(10): 1123-34.

16. Chávez-Gonzáles C, Soto TA. Evaluación del riesgo de síndrome de apnea obstructiva del sueño y somnolencia diurna utilizando el cuestionario de Berlín y las escalas Sleep Apnea Clinical Score y Epworth en pacientes con ronquido habitual atendidos en la consulta ambulatoria. Rev Chil Enfermedades Respir. 2018; 34(1): 19-27.

17. Baldini M, Chiapella MN, Fernández MA, Guardia S. STOP-Bang, una herramienta util y sencilla para el cribado del síndrome de apneas hipopneas obstructivas del sueño. Med B Aires. 2017; 77(3).

18. Rey de Castro J, Rosales-Mayor E, Weaver TE. Reliability and validity of the Functional Outcomes of Sleep Questionnaire - Spanish Short
Version (FOSQ-10SV) in peruvian patients With Obstructive Sleep Apnea. J Clin Sleep Med. 2018; 14(4): 615-21.

19. Pachauri Vallenas MC. Frecuencia del síndrome de apnea obstructiva del sueño en pacientes entre 18 a 85 años atendidos en la clínica odontológica de la universidad católica de Santa María, Arequipa 2013 [Tesis]. Arequipa: Universidad Católica Santa María. Facultad de Odontología; 2014.

20. Chung F, Yegneswaran B, Liao P, Chung SA, Vairavanathan S, Islam S, et al. STOP questionnaire: a tool to screen patients for obstructive sleep apnea. Anesthesiology. 2008; 108(5): 812-21.

21. Gómez Abanto JBD. Características clínico-epidemiológicas de pacientes con riesgo de síndrome de apnea-hipopnea obstructiva del sueño en un servicio de medicina interna de un hospital nacional de EsSalud, 2014 [Tesis]. Lima: Universidad de San Martín de Porres. Facultad de Medicina Humana; 2014.

\section{Correspondencia:}

Franca Maraís Sifuentes Peracchio Dirección: Jirón Bodegones 371 Santiago de Surco. Lima, Perú.

Teléfono: +51983549808

Correo electrónico: franca_sifuentes@usmp.pe

Recibido: 11 de enero de 2021

Evaluado: 01 de febrero de 2021 Aprobado: 15 de febrero de 2021

(c) La revista. Publicado por Universidad de San Martín de Porres, Perú. (cc) Br Licencia de Creative Commons Artículo en acceso abierto bajo términos de Licencia Creative Commons Atribución 4.0 Internacional. (http://creativecommons.org/licenses/by/4.0/)

ORCID iDs

Ricardo Andres Escobar Baigorria 1 https: / / orcid.org/0000-0001-5547-042X Franca Maraís Sifuentes Peracchio 10 https://orcid.org/0000-0002-6050-0598 Félix Llanos-Tejada 